TPeriodica Polytechnica Electrical Engineering and Computer Science

60(3), pp. 171-177, 2016

DOI: $10.3311 /$ PPee. 7160

Creative Commons Attribution (1)

RESEARCH ARTICLE

\section{Distributed RC One-Ports: Characteristic Functions and Their Relations}

\author{
Albin Szalai ${ }^{1 *}$, Vladimír Székely $^{1}$
}

Received 20 November 2013; accepted after revision 18 May 2016

\begin{abstract}
The present paper deals with the characteristic functions of distributed RC one-ports. It presents a detailed discussion about two characteristic functions of distributed $R C$ networks: the time-constant density function and the dipole intensity function. Based on the properties of these functions, their transformation schemes are presented. Distributed and lumped-element examples are shown as a demonstration of the practical operations of these schemes.

The paper also deals with the relation of these characteristic functions to the complex admittance, and introduces an identification method of RC one-ports in the admittance domain.
\end{abstract}

\section{Keywords}

distributed networks, RC networks, circuit theory

\footnotetext{
${ }^{1}$ Department of Electron Devices,

Faculty of Electrical Engineering and Informatics,

Budapest University of Technology and Economics,

H-1521 Budapest, P.O.B. 91, Hungary

*Corresponding author, e-mail: szalai@eet.bme.hu
}

\section{Introduction}

The investigation of RC one-ports has become a particularly important research area in the last two decades due to the emergence of thermal issues in integrated circuits. In order to achieve a better thermal design, it is essential to model its thermal behavior. From a more general point of view, it is an often encountered task to identify the structure of a network based on measurement, simulation and modeling (e.g. finding the poles and zeros of a one-port or transfer impedance, creating an equivalent circuit from time domain step response, etc.). In most cases, the main goal of this process is to obtain an accurate model of the measured system, or to identify the real physical structure based on its response [1]. This process may lead to the development of an effective non-destructive failure detection method [2] along with methods for optimizing the operating point [3].

In an earlier paper [4], we have demonstrated that a number of distributed RC networks cannot be described by poles and zeros; their distributed, "smeared" counterparts have to be used instead. In the case of RC networks, these extensions appear as two real-to-real functions, defined along the negative real axis of the complex plane. Let us refer to these functions as "characteristic functions".

The clarification of the relations between these characteristic functions is an anticipated demand, therefore it is the main subject of this paper. We have recognized that admittance-based complementary pairs can also be defined for these functions: these are introduced in the paper gradually.

The organization of the paper is as follows: the definition of the characteristic functions is presented in the next section, along with their relation to the port impedance. Section 3 deals with the connection of the two characteristic functions, deriving closed-form transformations between them. Examples demonstrating the operations of these transformation schemes are presented in Section 4. Section 5 introduces the admittancebased variants of the characteristic functions. The application of this variant for network identification is demonstrated in Section 6. Finally, Section 7 summarizes the results presented by the paper. 
All subsequent analysis is limited to the one-port behavior of passive, time-invariant and linear RC networks. The results are applicable to both distributed and lumped networks (although the primary goal of this work is the representation of distributed circuits).

\section{Definition of the Characteristic Functions}

Presuming that the aforementioned characteristic functions are not generally known, we consider it necessary to present here the short definition of these functions.

\subsection{The Time-Constant Spectrum}

The time-domain response of a linear RC network consists of many exponential functions [4] having different time-constant $(\tau)$ values. In the case of lumped circuits, the number of $\tau$ values occurring in the response is finite. In contrast, the distributed circuits have an infinite number of $\tau$ values. Let we say that the network has a spectrum of time-constants, and this spectrum characterizes the port impedance unambiguously.

If we apply a unit step current excitation to the port in the case of a lumped network (an example of what is shown in Fig. 1), the voltage response is as follows:

$$
u(t)=\sum_{i} R_{i}\left(1-\exp \left(-t / \tau_{i}\right)\right) .
$$

For further convenience, we introduce the following logarithmic variables $z=\log (t)$ and $\zeta=\log (\tau)$ :

$$
u(z)=\sum_{i} R_{i}\left(1-\exp \left(-\exp \left(z-\zeta_{i}\right)\right)\right)
$$

$R_{i}$ and $\zeta_{i}=\log \left(\tau_{i}\right)$ represent a spectrum consisting of discrete lines. However, in the case of distributed networks, the spectrum is continuous. In this case, the response can be described as

$$
u(z)=\int_{-\infty}^{\infty} R(\zeta) \cdot(1-\exp (-\exp (z-\zeta))) \mathrm{d} \zeta
$$

where $R(\zeta)$ is the time-constant spectrum expressed in logarithmic scale. This is the first characteristic function. $R(\zeta)$ can be generated using the following expression (if the function is negative, it has to be multiplied by -1 ) (proof in [4]):

$$
R(\zeta)=\frac{1}{\pi} \operatorname{Im}\{\mathbf{Z}(\mathbf{s}=-\exp (\zeta))\}
$$

$\mathbf{Z}(\mathbf{s})$ is the complex impedance function of the port. The inverse operation can be performed as well. The impedance can be calculated if $R(\zeta)$ is known [4]:

$$
\mathbf{Z}(\mathbf{S})=\int_{-\infty}^{\infty} \frac{R(-x)}{1+\exp (\mathbf{S}-x)} \mathrm{d} x
$$

where $\mathbf{S}=\log (\mathbf{s})$ is the natural logarithm of the complex frequency.

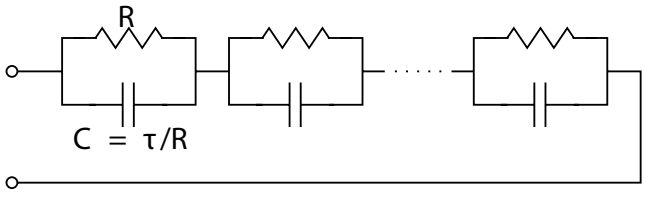

Fig. 1 RC one-port, example (Foster's first canonical form)

\subsection{The Dipole Intensity Function}

It is a well-known fact [4] that in the case of a lumped RC network, the poles and zeros of the port impedance alternate along the $-\sigma$ axis, and the sequence of the poles and zeros begins with a pole [5] (see Fig. 2). The adjacent poles and zeros either cancel each other out or let each other be effective. Let us see Fig. 3. If the first pole is followed closely by the first zero, the first pole is canceled. On the contrary, if the first zero is placed closely to the next pole, the first pole is effective. The essential information is carried by the relative position of the zeros between two neighboring poles. A possible interpretation is that the pairs of adjacent poles and zeros constitute a dipole. The intensity of that dipole depends on the distance between the pole and the zero.

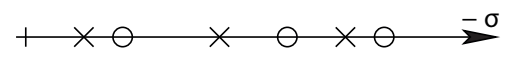

Fig. 2 Pole-zero arrangement of an RC one-port

In order to construct the definition of the dipole intensity function, we first introduce a logarithmic variable on the negative $\sigma$ axis:

$$
\Sigma=\log (-\sigma)
$$

Let us investigate a $\Delta \Sigma$ interval of the logarithmic $\Sigma$ axis bounded by two adjacent poles (see Fig. 3).

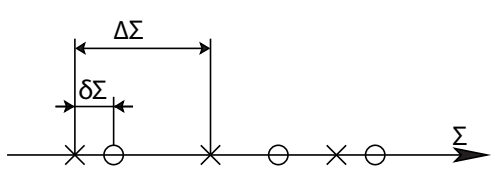

Fig. 3 To the definition of dipole intensity

The distance between the left-hand pole and the inner zero is $\delta \Sigma$. Suppose that the density of the poles tends to infinity. In other words, $\Delta \Sigma$ becomes infinitely small. In this case, the dipole intensity function is

$$
I_{d}(\Sigma)=\lim _{\Delta \Sigma \rightarrow 0} \frac{\delta \Sigma}{\Delta \Sigma}
$$

This is the second characteristic function.

Considering that the poles and zeros of an RC port impedance alternate, it directly follows that

$$
0 \leq I_{d} \leq 1 .
$$


$I_{d}(\Sigma)$ can be generated using the following expressions (if the function is negative, it has to be multiplied by -1) (proof in [4]):

$$
\begin{gathered}
I_{d}(\Sigma)=\frac{1}{\pi} \operatorname{Im}\{\log \mathbf{Z}(\mathbf{s}=-\exp (\Sigma))\} \\
I_{d}(\Sigma)=\frac{1}{\pi} \arg (\mathbf{Z}(\mathbf{s}=-\exp (\Sigma))) .
\end{gathered}
$$

$\mathbf{Z}(\mathbf{s})$ is again the complex impedance function of the port. The inverse operation can be performed as well. The impedance can be calculated if $I_{d}(\Sigma)$ is known:

$$
\log (\mathbf{Z}(\mathbf{S}))=\log \left(R_{0}\right)-\int_{-\infty}^{\infty} I_{d}(x) \frac{\exp (\mathbf{S}-x)}{1+\exp (\mathbf{S}-x)} \mathrm{d} x
$$

\section{Relation Between the Characteristic Functions}

As we have seen in the former section, the properties of a passive, linear, evenly distributed RC one-port can be described by either the $R(\zeta) \tau$-spectrum or the $I_{d}(\Sigma)$ dipole intensity function.

Since both descriptions are unambiguous and complete, one of them must determine the other. Consequently, there must be a way to calculate one from the other. This requirement holds theoretical significance, since the existence of an unambiguous mapping between the two representative functions is a strict expectation. Practical benefits of this algorithmic procedure can be expected, since the need to calculate $I_{d}(\Sigma)$ knowing $R(\zeta)$ or vice versa may arise. This is why we have elaborated and hereby present the $R(\zeta) \leftrightarrow I_{d}(\Sigma)$ transformation schemes:

$$
\begin{gathered}
I_{d}(\Sigma)=\frac{1}{\pi} \arg \int_{-\infty}^{\infty} \frac{R(-x)}{1-\exp (\Sigma-x)} \mathrm{d} x \\
R(\Sigma)=\frac{1}{\pi} R_{0} \cdot \operatorname{Im}\left\{\exp \left(\int_{-\infty}^{\infty} I_{d}(x) \frac{\exp (\Sigma-x)}{1-\exp (\Sigma-x)} \mathrm{d} x\right)\right\}
\end{gathered}
$$

$R_{0}$ is the resistance at zero frequency. Proof of these two equations can be found in Appendix A.

Both equations can be rearranged to obtain the convolution forms as well:

$$
\begin{gathered}
I_{d}(x)=\frac{1}{\pi} \arg \left(R_{M}(x) \otimes \frac{1}{1-\exp (x)}\right) \\
R_{M}(x)=\frac{1}{\pi} R_{0} \cdot \operatorname{Im}\left\{\exp \left(I_{d}(x) \otimes \frac{\exp (x)}{1-\exp (x)}\right)\right\}
\end{gathered}
$$

where $R_{M}(x)=R(-x)$ is the mirrored $\tau$-spectrum function.

It is clear that these relations are non-linear due to the properties of the functions $\arg ()$ and $\exp ()$. The convolution step is linear; thus the transformations consist of a linear step followed by a non-linear one.

During the evaluation of the Eqs. (12) (13), we encounter difficulties. These integrals are improper, since the integrand has a discontinuity if the denominator is zero. Fortunately, closed-form integral of $1 /(1-\exp (x))$ exists, and in the region of discontinuity, the principal value theory of Cauchy [6] is applicable. Another way of practical calculation is presented by the below formula:

$$
I_{d}(\Sigma)=\lim _{\delta \rightarrow 0} \frac{1}{\pi-\delta} \arg \int_{-\infty}^{\infty} \frac{R(-x)}{1-\exp (\Sigma+\jmath \delta-x)} \mathrm{d} x
$$

The error introduced by this approximation is equivalent to the result of the convolution by a narrow pulse [7]

$$
e(z)=\frac{1}{\pi-\delta} \frac{\sin \delta \cdot \exp (z)}{1-2 \cos \delta \exp (z)+\exp (2 z)}
$$

The integral of this pulse function is unity, whereas its halfvalue width is approximately $2 \delta$.

A similar "maneuver" can be applied to Eq. (13) as well.

\section{Examples for the $R(\zeta) \leftrightarrow I_{d}(\Sigma)$ transformation}

In order to verify the results of the former section, we have chosen a structure with known analytic characteristic functions. It is a distributed thermal RC structure: the three-dimensional heat transfer in a semi-infinite medium, see Fig. 4. The investigated structure was described in our earlier paper [8]. The "input port" is a hemisphere of radius $r_{0}$. Using the analytic solution of the problem [9], the one-port impedance for this port can be described as

$$
\mathbf{Z}(\mathbf{s})=\frac{1}{2 \pi \lambda r_{0}} \frac{1}{1+r_{0} \sqrt{\mathbf{s c} / \lambda}}
$$

where $\lambda$ and $c$ are the heat conductivity and the heat capacitance per unit volume, respectively.

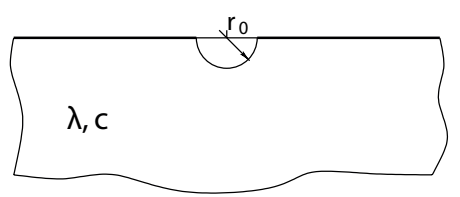

Fig. 4 The distributed thermal RC circuit

Using Eqs. (4) and (10), the two characteristic functions can be calculated as

$$
R(\sigma)=\frac{1}{2 \pi^{2}} \frac{1}{\lambda} \frac{\sqrt{\sigma c / \lambda}}{1+r_{0}^{2} \sigma c / \lambda}
$$




$$
I_{d}(\sigma)=\frac{1}{\pi} \arctan \left(r_{0} \sqrt{c / \lambda} \sqrt{\sigma}\right)
$$

First, the $R(\zeta) \rightarrow I_{d}(\Sigma)$ direction was tested. The integral expression of (12) was calculated using numerical approximation. The applied sampling rate was 0.02 . The discontinuity of the integrand was avoided by choosing $\delta=0.02$. The resulting function is plotted in Fig. 5 along with the analytic one. It is obvious that the agreement between the analytic and calculated curves is excellent, with the exception of the $\Sigma>30$ region. Error in the latter region is caused by the finite accuracy of the numerical calculation of the integral.

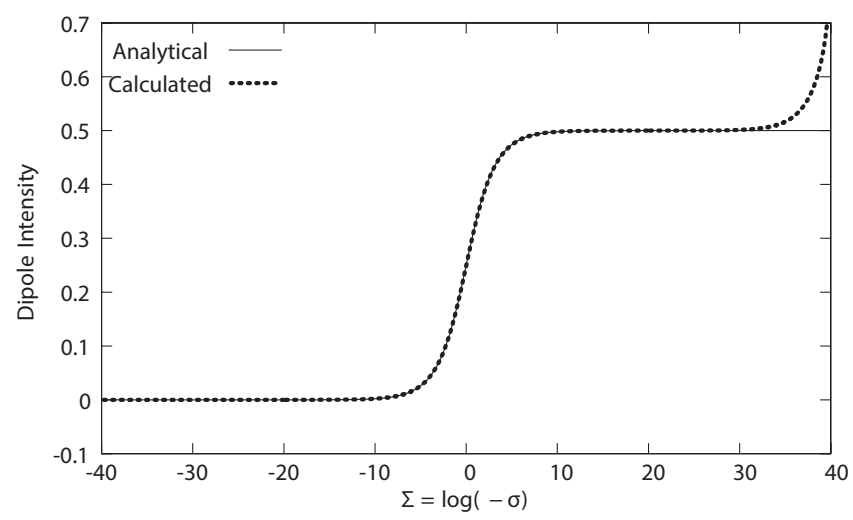

Fig. 5 Result of the $R(\zeta) \rightarrow I_{d}(\Sigma)$ transformation. Dots have been calculated using (12); the continuous line represents the "true" function

In the second example, the $I_{d}(\Sigma) \rightarrow R(\zeta)$ transformation was tested. The problem investigated in the first test was addressed here as well. We intended to calculate (19) in knowledge of (20). The integral expression of (13) was calculated using numerical approximation again. The applied sampling rate was 0.02 , with $\delta=0.02$. Figure 6 presents the result of the calculation along with the known analytical function. The agreement is excellent in this case as well.

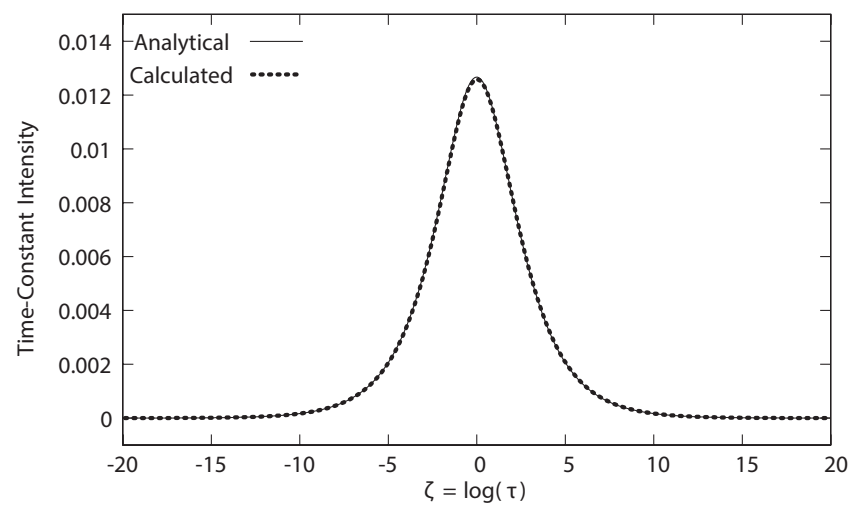

Fig. 6 Result of the $I_{d}(\Sigma) \rightarrow R(\zeta)$ transformation

Although the characteristic functions $R(\zeta)$ and $I_{d}(\Sigma)$ are intended to be used for distributed networks, they can be applied to lumped circuits as well. In this case, the $R(\zeta)$ spectrum consists of distinct lines only. The $I_{d}(\Sigma)$ dipole intensity is similar to a telegraph signal: the zero-to-one transitions stand for poles, while one-to-zero transitions stand for zeros [4].

The transformations $I_{d}(\Sigma) \leftrightarrow R(\zeta)$ must work in the case of lumped element networks as well. Examples that prove this statement are presented in Appendix B.

\section{Characteristic Functions Related to the Complex Admittance}

We have so far discussed the properties and relations of $R(\zeta)$ and $I_{d}(\Sigma)$ characteristic functions. For the sake of completeness, we need to mention that a complementary pair for each of these functions can be defined as well, based on the $\mathbf{Y}(\mathbf{s})$ one-port admittance. In this section, we will introduce these characteristic functions.

The admittance-based description can be generated easily, using the second Foster canonical form shown in Fig. 7. Using the logarithmic variables $\mathbf{S}=\log (\mathbf{s})$ and $\zeta=\log (\tau)$, the admittance of a single stage can be described as

$$
\mathbf{Y}=\frac{G}{1+\exp (-\mathbf{S}-\zeta)}
$$

Supposing that the distributed network has a continuous spectrum of time constants

$$
\mathbf{Y}(\mathbf{S})=\int_{-\infty}^{\infty} \frac{G(\zeta)}{1+\exp (-\mathbf{S}-\zeta)} \mathrm{d} \zeta
$$

Now we apply (22) along a line of the complex plane, which is very close to, but does not coincide with the negative real axis (the integration path and the axis form an angle $\varepsilon$ ):

$$
\begin{gathered}
\mathbf{s}=-(1+j \varepsilon) \exp (\Sigma) \\
\exp (-\mathbf{S})=\frac{1}{\mathbf{s}}=\frac{1}{-(1+j \delta) \exp (\Sigma)} \\
\mathbf{Y}(\Sigma)=\int_{-\infty}^{\infty} \frac{G(-x)}{1-1 /(1+j \varepsilon) \exp (-\Sigma+x)} \mathrm{d} x \\
\mathbf{Y}(x)=G(-x) \otimes \frac{1}{1-\frac{\exp (-x)}{(1+j \varepsilon)}}=G(-x) \otimes \mathbf{W}(x) \\
\operatorname{Im}\{W(x)\}=\frac{-\delta \exp (x)}{(1-\exp (x))^{2}+\delta^{2} \exp (x)^{2}}
\end{gathered}
$$

[4] proves that

$$
\lim _{\delta \rightarrow \infty} \operatorname{Im}\{\mathbf{W}(x)\}=-\pi \delta(x)
$$

$$
G(\zeta=-x)=\frac{1}{\pi} \operatorname{Im}\{\mathbf{Y}(\mathbf{s}=-\exp (x))\}
$$


The $G(\zeta)$ function is the time-constant spectrum in the admittance-based approach.

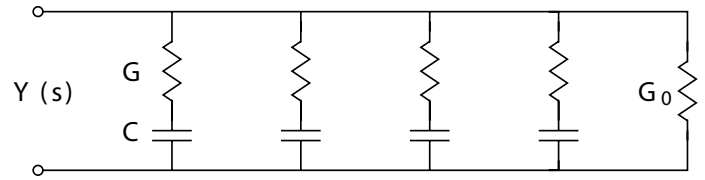

Fig. 7 Foster II canonical form of an RC one-port

Next, we derive the Y-counterpart of Eq. (11). Substituting $\mathbf{Z}(\mathbf{s})=1 / \mathbf{Y}(\mathbf{s})$ we have found that

$$
\begin{aligned}
\log \left(\frac{1}{\mathbf{Y}(\mathbf{S})}\right) & =-\log (\mathbf{Y}(\mathbf{S})) \\
& =\log \left(R_{0}\right)-\int_{-\infty}^{\infty} I_{d}(x) \frac{\exp (\mathbf{S}-x)}{1+\exp (\mathbf{S}-x)} \mathrm{d} x \\
\log (\mathbf{Y}(\mathbf{S}))= & \log \left(G_{0}\right)-\int_{-\infty}^{\infty}-I_{d Y}(x) \frac{\exp (\mathbf{S}-x)}{1+\exp (\mathbf{S}-x)} \mathrm{d} x
\end{aligned}
$$

where $I_{d Y}$ is the dipole intensity for admittance, and $G_{0}$ is the DC conductance of the network. It is important to recognize that $I_{d Y}=-I_{d}$ thus, the admittance-based dipole intensity function and the impedance-based dipole intensity function differ only in their signs.

The time-domain behavior is important as well; it is also necessary to be investigated. Equation (22) will be converted into time domain using the inverse Laplace transform.

$$
\mathbf{Y}(\mathbf{s})=\int_{-\infty}^{\infty} \frac{G(-x)}{1+\exp (-x) / \mathbf{s}} \mathrm{d} x
$$

Let the voltage excitation be a $u=1(t)$ unit-step function with $1 / \mathbf{s}$ Laplace transform. The current response is

$$
\begin{gathered}
\mathbf{I}(\mathbf{s})=\mathbf{Y}(\mathbf{s}) \cdot \frac{1}{\mathbf{s}}=\int_{-\infty}^{\infty} \frac{G(-x)}{\mathbf{s}+\exp (-x)} \mathrm{d} x \\
i(t)=\int_{-\infty}^{\infty} G(-x) \cdot \exp (-t / \exp (-x)) \mathrm{d} x
\end{gathered}
$$

Observing this equation we can state that the current response is the totality of components showing exponential decay and $G$ is the spectrum of these exponents.

\section{Measurement and Identification of RC One-Ports in the Admittance Domain}

The NID (Network Identification by Deconvolution) method [10], a deconvolution based approach, has become widely applied in the last two decades for the identification of RC oneports. This method operates in the impedance domain, using the voltage response of the current unit-step excitation of the examined network. The key equation of this procedure is a direct consequence of Eq. (3):

$$
\frac{\mathrm{d} u}{\mathrm{~d} z}=R(z) \otimes \exp (z-\exp (z))
$$

Possessing the measured $u(z)$ function, a numerical derivation step and a deconvolution operation lead to the $R(z)$ time-constant spectrum [11]. The method is widely used for the preparation of heat-flow maps of thermal structures such as IC packages, heat sinks etc. [12]. In this case, the analogy between thermal and electrical conduction is exploited. According to the usual modeling rules - used in widespread modeling methods voltage represents temperature and current represents heat flux.

It seems straightforward to define the admittance-based version of the method as well. In this case, a voltage step excitation is applied and the current response is measured. Here we use the GC equivalent of our initial RC network, which has serial capacitance and conductance inserted in each of its parallel-coupled stages (see Fig. 8). The response of the network can be described by using (34) as

$$
i(z)=\int_{-\infty}^{\infty} G(-x) \cdot \exp (-\exp (z+x)) \mathrm{d} x
$$

where $t$ is substituted by $\exp (z)$. Deriving both sides by $z$, results in

$$
\begin{gathered}
\frac{\mathrm{d} i}{\mathrm{~d} z}=-\int_{-\infty}^{\infty} G(-x) \exp (-\exp (z+x)) \cdot \exp (z+x) \mathrm{d} x \\
\frac{\mathrm{d} i}{\mathrm{~d} z}=-G(z) \otimes \exp (z-\exp (z))
\end{gathered}
$$

If we measure the $i(t)$ response to a unit step voltage excitation, we are able to calculate its time-constant spectrum $G(z)$. The procedure is similar to the case of Eq. (35).
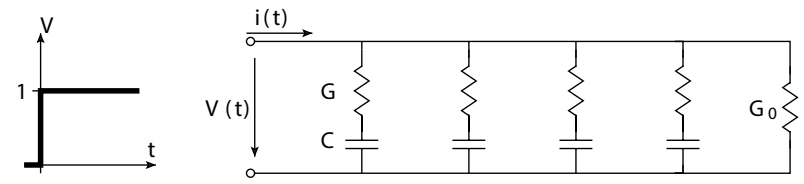

Fig. 8 To the admittance-based version of the NID method

The advantage of this identification process is that it results in an easier measurement setup for pure electrical networks. Sometimes it is more simple to create an accurate voltage step function than a current step function. Of course this method is not a viable option for the investigation of thermal networks, since it is difficult to realize a temperature step on thermal $\mathrm{RC}$ structures.

\section{Conclusions}

The paper presents additions to the theory of distributed $\mathrm{RC}$ one-ports published previously by one of the authors. Closed-form relations between the characteristic functions (time-constant spectrum, dipole intensity) have been derived. 
The admittance-based counterparts of the characteristic functions have been introduced. A variant method of network identification by deconvolution (using voltage step excitation and recording the current response) is elaborated.

\section{A Appendix}

\section{A.1 Proof of Eq. (12)}

In Eq. (10) we substitute $\mathbf{Z}(\mathbf{s})$ impedance function with (5), taking into account the following:

- Using (10) we write $-\exp (\Sigma)$ instead of $\mathbf{s}$ since we follow the negative real axis and $\Sigma$ is logarithmic,

- $\mathbf{S}=\log (\mathbf{s})$, thus in place of $\mathbf{S}$ we write $\log (-\exp (\Sigma))=$ $\Sigma+j \pi$. Therefore

$$
\begin{gathered}
I_{d}(\Sigma)=\frac{1}{\pi} \arg \int_{-\infty}^{\infty} \frac{R(-x)}{1+\exp (\Sigma+j \pi-x)} d x \\
I_{d}(\Sigma)=\frac{1}{\pi} \arg \int_{-\infty}^{\infty} \frac{R(-x)}{1-\exp (\Sigma-x)} d x
\end{gathered}
$$

\section{A.2 Proof of Eq. (13)}

The calculation starts with Eq. (11). As a first step the exponential function of both sides is calculated:

$$
\begin{aligned}
\exp (\log (\mathbf{Z}(\mathbf{s}))) & =(\mathbf{Z}(\mathbf{S})) \\
& =R_{0} \cdot \exp \left(\int_{-\infty}^{\infty}-I_{d}(x) \frac{\exp (\mathbf{S}-x)}{1+\exp (\mathbf{S}-x)} \mathrm{d} x\right)
\end{aligned}
$$

Substituting $\mathbf{S}=\Sigma+j \pi$ we get

$$
\mathbf{Z}(\Sigma)=R_{0} \cdot \exp \left(\int_{-\infty}^{\infty}-I_{d}(x) \frac{\exp (\Sigma+j \pi-x)}{1+\exp (\Sigma+j \pi-x)} d x\right)
$$

After inserting this equation into (4), we found that

$$
R(\Sigma)=\frac{1}{\pi} \operatorname{Im}\left\{R_{0} \exp \left(\int_{-\infty}^{\infty}-I_{d}(x) \frac{-\exp (\Sigma-x)}{1-\exp (\Sigma-x)}\right)\right\}
$$

Following some mathematical rearrangements, we have

$$
R(\Sigma)=\frac{1}{\pi} R_{0} \cdot \operatorname{Im}\left\{\exp \left(\int_{-\infty}^{\infty} I_{d}(x) \frac{\exp (\Sigma-x)}{1-\exp (\Sigma-x)} d x\right)\right\}
$$

\section{B Examples for the Lumped Circuit Case}

In the case of a lumped element network, the integrals in the (12) and (13) transformation relations can be substituted by summation. The equation (12) can be written as

$$
I_{d}(\Sigma)=\lim _{\delta \rightarrow \infty} \frac{1}{\pi-\delta} \arg \sum_{i} \frac{R_{i}}{1-\exp \left(\Sigma+j \delta-x_{i}\right)}
$$

where $R_{i}, \tau_{i}$ are discrete lines of the $\tau$-spectrum and $x_{i}=$ $\log \left(1 / \tau_{i}\right)=-\log \left(\tau_{i}\right)$.
This appendix demonstrates that (12) and (13) transformations are "well applicable" in the case of lumped element networks as well. The investigated lumped element network consists of four pairs of RC elements. The example network is described in our earlier paper [8]. The element values can be found in Table 1. These values determine the time-constant spectrum of the example. Each RC element creates a single spectrum line with an amplitude of $R_{i}$ at the $\tau_{i}$ position. Applying the (45) transformation formula we obtain the dipole intensity function of the network. The calculated result is plotted in Fig. 10 along with the analytical function. The applied sampling rate was 0.02 with $\delta=0.002$. The $I_{d}(\Sigma) \leftrightarrow R(\zeta)$ transformation has also been tested. The problem investigated in the first test was addressed here as well. We intended to calculate the time-constant spectrum in knowledge of the dipole intensity function. The integral expression of (13) was evaluated using numerical approximation again. The applied sampling rate was 0.02 , with $\delta=0.02$. Figure 11 presents the result of the calculation along with the known analytic function. The agreement is excellent in this case as well.

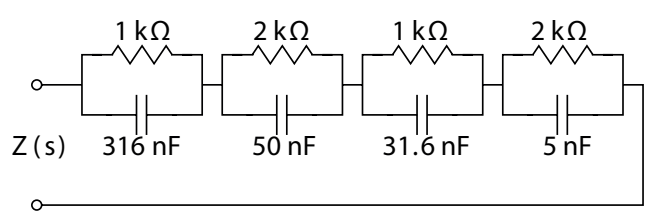

Fig. 9 Lumped RC one-port

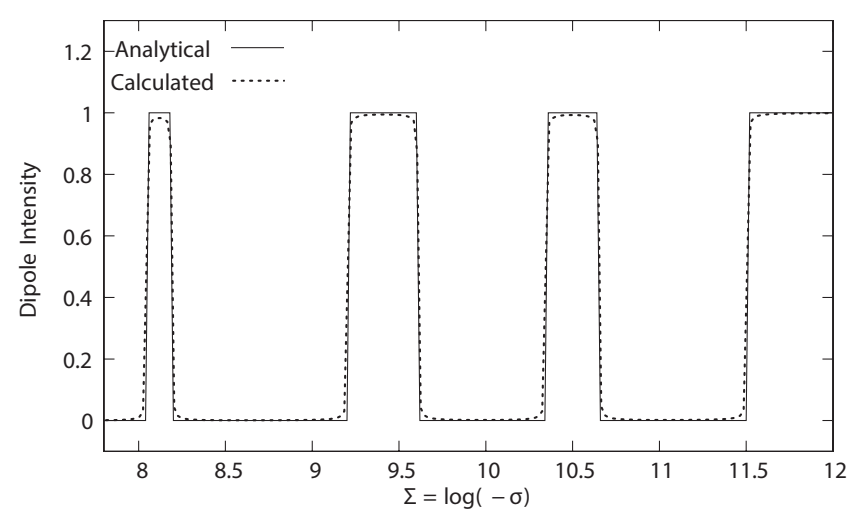

Fig. 10 Dipole intensity function of the one-port shown in Fig. 9

Table 1 Lumped Element Network Parameters

\begin{tabular}{lllll}
\hline$\tau[\mu s]$ & 316 & 100 & 31.6 & 10 \\
\hline $\begin{array}{l}\text { Amplitude } \\
{[k \Omega]}\end{array}$ & 1 & 2 & 1 & 2 \\
\hline
\end{tabular}




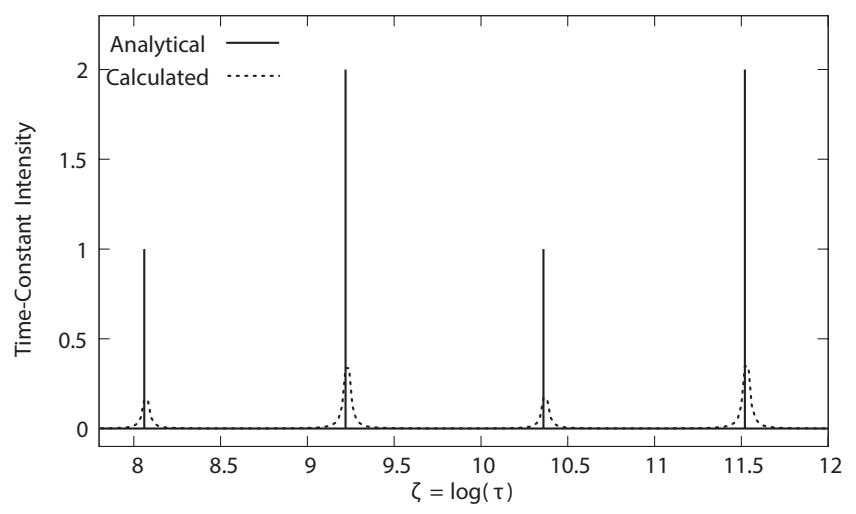

Fig. 11 Time-constant spectrum of the one-port shown in Fig. 9. The four peaks of the calculated result approximate the four Dirac- $\delta$ functions, their integrals are very close to the 1-2-1-2 amplitudes.

\section{References}

[1] Ender, F., Hantos, G., Schweitzer, D., Szabo, P. G. "Thermal characterization of multichip structures." In: Thermal Investigations of ICs and Systems (THERMINIC), 2013 19th International Workshop on, Berlin, Sept. 25-27, 2013, pp. 319-322. DOI: 10.1109/THERMINIC.2013.6675241

[2] Kim, H.-H., Choi, S.-H., Shin, S.-H., Lee, Y.-K., Choi, S.-M., Yi, S. "Thermal transient characteristics of die attach in high power LED PKG." Microelectronics Reliability. 48(3), pp. 445-454. 2008.

DOI: 10.1016/j.microrel.2007.08.009

[3] Lasance, C. J. M., Poppe, A. (eds.) "Thermal Management for LED Applications." Springer-Verlag New York, 2014.

DOI: $10.1007 / 978-1-4614-5091-7$

[4] Szekely, V. "On the representation of infinite-length distributed RC oneports." IEEE Transactions on Circuits and Systems. 38(7), pp. 711-719. 1991. DOI: 10.1109/31.135743

[5] Bode, H. W. "Network Analysis and Feedback Amplier Design." D. Van Nostrand, Princeton, 1945.

[6] Bronstein, I. N., Semendyayev, K. A. "Handbook of Mathematics." 3rd edition. Springer-Verlag, 1997.

[7] Chen, W.-K. (ed.) "The Circuits and Filters Handbook." 3rd edition, Volume Feedback, Nonlinearm and Distributed Circuits, Chapter 18. CRC Press, 2009.

[8] Szekely, V. "Convolution Calculus in the Network Theory and Identication." In: Proceedings of ECCTD'97, Budapest, 1997, pp. 49-56.

[9] Carslaw, H. S., Jaeger, J. C. "Conduction of Heat in Solids." Oxford University Press, Oxford, 1959.

[10] Szekely, V. "Identification of RC networks by deconvolution: chances and limits." IEEE Transactions on Circuits and Systems I: Fundamental Theory and Applications. 45(3), pp. 244-258. 1998.

DOI: $10.1109 / 81.662698$

[11] Cohn-Sfetcu, S., Smith, M. R., Nichols, S. T., Henry, D. L. "A digital technique for analyzing a class of multicomponent signals." Proceedings of the IEEE. 63(10), pp. 1460-1467. 1975.

[12] Szekely, V., Van Bien, T. "Fine structure of heat flow path in semiconductor devices: a measurement and identication method." Solid-State Electronics. 31(9), pp. 1363-1368. 1988.

DOI: $10.1016 / 0038-1101(88) 90099-8$ 